\title{
Review Article \\ Manipulation of Innate and Adaptive Immunity through Cancer Vaccines
}

\author{
Elias J. Sayour and Duane A. Mitchell \\ UF Brain Tumor Immunotherapy Program, Preston A. Wells Jr. Center for Brain Tumor Therapy, \\ Department of Neurosurgery, University of Florida, Gainesville, FL, USA
}

Correspondence should be addressed to Elias J. Sayour; esayour@ufl.edu

Received 2 December 2016; Accepted 4 January 2017; Published 6 February 2017

Academic Editor: Said Dermime

Copyright ( 2017 Elias J. Sayour and Duane A. Mitchell. This is an open access article distributed under the Creative Commons Attribution License, which permits unrestricted use, distribution, and reproduction in any medium, provided the original work is properly cited.

\begin{abstract}
Although cancer immunotherapy has shown significant promise in mediating efficacious responses, it remains encumbered by tumor heterogeneity, loss of tumor-specific antigen targets, and the regulatory milieu both regionally and systemically. Cross talk between the innate and adaptive immune response may be requisite to polarize sustained antigen specific immunity. Cancer vaccines can serve as an essential fulcrum in initiating innate immunity while molding and sustaining adaptive immunity. Although peptide vaccines have shown tepid responses in a therapeutic setting with poor correlates for immune activity, RNA vaccines activate innate immune responses and have shown promising effects in preclinical and clinical studies based on enhanced DC migration. While the mechanistic insights behind the interplay between innate and adaptive immunity may be unique to the immunotherapeutic being investigated, understanding this dynamic is important to coordinate the different arms of the immune response in a focused response against cancer antigens.
\end{abstract}

\section{Introduction}

Immune targeting of cancer antigens has been employed since the original experiments of William H. Coley in the late 1800s [1]. In 1891, Coley administered streptococcal organisms into an inoperable patient and was able to demonstrate tumor regression [1]. He later injected over a thousand cancer patients with bacterial products (i.e., Streptococcus pyogenes, Serratia marcescens known as Coley's toxins) and demonstrated promising results in both bone and soft tissue sarcomas $[1,2]$. Coley's experiments utilized toll-like receptor (TLR) agonists in the bacterial products to harness the innate immune response [2]. Now, over a century later, cancer immunotherapy is a burgeoning field and has elicited statistically significant survival benefits in patients with refractory malignancies [3-5]. With the advent of sipuleucel-T and ipilimumab, the first FDA approved immunotherapeutics have arrived and the immune correlates for successful intervention are slowly being unraveled [3-5]. However, while cancer immunotherapy has shown significant promise in mediating efficacious response, it remains encumbered by tumor heterogeneity, loss of tumor-specific antigen targets, and the regulatory milieu both regionally and systemically [6-9]. Cancer vaccines have especially lagged behind checkpoint blockers and adoptive cellular therapy (ACT) in mediating robust antitumor immunity $[10,11]$.

Cancer vaccines deliver tumor antigens in the form of novel mutated epitopes, viral epitopes, developmental antigens, or self-differentiation antigens to endogenous innate cells (i.e., antigen presenting cells) [12-16]. Antigen presenting cells (APCs) become activated in the presence of vaccine carrying antigen, typically accompanied by adjuvants that provide the necessary inflammatory cues [17]. APCs then process the vaccine's tumor antigens into epitopes, which they present on the surface of their MHC class I and class II molecules for presentation to CD4 and CD8+ T cells [17]. They subsequently traffic to local draining lymph nodes, engaging, activating, and redirecting $\mathrm{T}$ cells to reject the presented tumor epitopes [17]. For a cancer vaccine to be effective, APC activation, trafficking, and T cell priming are essential initial steps. Secondary steps such as T cell proliferation, trafficking, tumor extravasation, and engagement 
of tumor antigens are also essential. In this review, we will examine promising strategies employed to enhance the initial steps of effective cancer vaccination followed by discussion of their synergistic roles with immune checkpoints to potentiate requisite secondary steps.

\section{Initiating Innate Immunity}

The innate immune system, as one of the first lines of defense, must recognize danger signals and respond accordingly [18]. Although $\mathrm{T}$ cells are the hallmark of an effective cancer immunotherapeutic response, they are often ineffective without activation of the innate immune system [10]. Innate immune cells such as natural killer cells, neutrophils, and macrophages have been shown to mediate regression in resistant murine models and can be harnessed in conjunction with adoptive $\mathrm{T}$ cell strategies [10, 19-21]. As opposed to discriminating between self and nonself, the danger theory purports that the innate immune system is primarily activated in response to danger signals [22, 23]. Pattern recognition receptors (PRRs) (i.e., endosomal TLRs and cytosolic sensors such as retinoic acid inducible gene I (RIGI) and melanoma differentiation-associated protein 5 (MDA5)) within innate immune cells are vital for perceiving danger signals (i.e., pathogen associated molecular patterns (PAMPs) and damage associated molecular patterns (DAMPs)) released from affected cells [18, 24, 25]. Since the kinetics of the immune response are sensitive to danger signals, methods to exploit this sensitivity may potentiate adaptive immunity [26]. This has been routinely employed through the use of chemotherapy such as cyclophosphamide and fludarabine in conjunction with promising $\mathrm{T}$ cell therapies including adoptively transferred tumor infiltrating lymphocytes (TILs) and chimeric antigen receptor (CAR) modified T cells [10, 27-30]. Although lymphodepletion with cyclophosphamide and fludarabine might be expected to mitigate against potent $\mathrm{T}$ cell responses, these agents have been associated with more potent cellular immunotherapy [31]. These enhancing effects are attributed not only to removal of regulatory compartments such as regulatory $\mathrm{T}$ cells (i.e., FoxP3+CD25+ T cells) and myeloid derived suppressor cell populations but also to released DAMPs which assist in engendering the inflammatory niche necessary to sustain the survival of adoptively transferred antigen specific T cells [31-35]. Although host-conditioning may be necessary to potentiate adoptive cell therapy, its implementation conflicts with immunotherapy's promise of delivering targeted agents without deleterious effects. Alternatively, development of experimental therapies that leverage both innate and adaptive immune arms without illicit effect is more attractive [36]. While synergy between innate and adaptive immunity is expected to potentiate immunotherapeutic response, chronic inflammatory stimuli may stymie an effective adaptive response [37-40]. In preclinical studies, low-dose infection with intradermal Trypanosoma congolense mediated expansion of regulatory T cells [37-40]. Similarly, as malignancies grow, they may precipitate similar levels of inflammation that predispose induction of regulatory cell subsets, which ultimately confound an appropriate adaptive response $[34,41$,
42]. These inadequate innate responses predispose formation of regulatory cells that usurp effector T cells [34, 41, 42].

However, redirecting the innate immune system has become tenable locally (at the tumor site) with oncolytic viruses $[43,44]$. Oncolytic viruses can be native or attenuated and be preferentially harnessed against cancer cells $[43,44]$. Since cancer cells contain low levels of protein kinase $\mathrm{R}$ (regulates abnormal cell proliferation and antiviral response), oncolytic viruses can preferentially replicate within malignant tumors [43-45]. Preferential transfection and replication within tumor cells mediate their direct lysis, releasing both soluble tumor antigens and DAMPs which lead toward a broader systemic response against the tumor [43]. Similar to oncolytic viruses, cancer vaccines can be harnessed to incite an innate response against select tumor antigens but they bypass the complexity of intratumoral oncolytic viral administration by activating innate immunity peripherally.

\section{Cancer Vaccines}

Cancer vaccines can be harnessed to educate the senescent immune system against tumor antigens. DAMPs are released after local vaccine injection, allowing APCs to traffic, pick up tumor antigens, and migrate to local draining lymph nodes where they present their antigens to T cells [46]. Challenges remain though in identifying the optimal source of antigen for cancer vaccines. Differentiation antigens such as cancer testis antigens have been employed as attractive immunotherapeutic targets based on strong immunogenicity with scant expression on normal tissues [47-49]. Exclusively tumorspecific mutated antigens, while more attractive, are few in number and may not be uniformly expressed across tumors or within an individual tumor $[7,50,51]$. After identification of the target antigen, cancer vaccines must deliver tumor antigens and have been employed to do so in the form of whole tumor lysate, peptides, or nucleic acids [17]. Whole tumor lysate is limited from difficult to access tumors, may include self-antigens, and have been shown to result in poor APC uptake, inadequate antigen cross-presentation, and tepid CD8+ T cell responses $[52,53]$. Alternatively, peptide vaccines can be constructed from the most immunogenic or cancer specific epitopes, but since peptides are not sufficiently antigenic, they are typically accompanied by an adjuvant (i.e., GM-CSF, KLH) [54]. Examples of cancer peptide vaccines that have been studied in phase III trials include the MAGEA3 cancer testis antigen for non-small cell squamous lung cancer/melanoma, rindopepimut for glioblastoma (GBM), and sipuleucel-T for prostate cancer [5, 7, 48, 51]. While cancer testis antigens are promising therapeutic targets, in two phase III trials for non-small cell lung cancer (NSCLC) and melanoma, MAGE-A3 (fusion protein administered with immunostimulant AS15) failed to extend disease-free survival [48]. Similarly, rindopepimut (peptide spanning junction of the tumor-specific EGFRVIII mutation found in 30-40\% of GBM patients) failed to meet its prespecified end-point in a randomized phase III study [7, 54]. Sipuleucel-T (a cell based peptide vaccine) activates immature DCs ex vivo using a fusion protein containing prostatic acid phosphatase coupled to GM-CSF and has shown efficacy by improving overall 
survival in metastatic castration-resistant prostate cancer but remains encumbered by significant cost/complexity, which has prevented its widespread adoption $[5,55]$. Meanwhile, the immune correlates of successful vaccination have yet to be fully unraveled [55]. In the case of sipuleucel-T, T cell responses were elevated in the presence of antigen conjugated to GM-CSF and were not as robust using unconjugated antigens highlighting the need for further investigation [55].

Despite limited progress in the setting of therapeutic vaccines, preventative vaccines such as the prophylactic HPV vaccine (Human Papillomavirus 9-valent vaccine, recombinant) have been utilized to avert cervical cancer; however, these vaccines do not induce strong therapeutic responses against established HPV lesions and active infections [56]. While cancers that are virally propagated (i.e., EBV+ Burkitt's disease, hepatocellular carcinoma) may benefit from prophylactic vaccines, new paradigms for therapeutic vaccines need to be established.

Peptide vaccines continue to be mired by local administration of poorly immunogenic antigens [11]. Moreover, peptide vaccines remain limited by $\mathrm{MHC}$ class restriction often constraining trials to HLA A2 selected patients (limiting accrual for trials with limited patient numbers (i.e., pediatric malignancies) $[57,58]$. Alternatively, nucleic acids allow for a patient's intracellular machinery to translate and process tumor encoding transcripts based on an individual specific HLA haplotype and can thus be leveraged for the population at large [58-60]. Nucleic acids encoding for tumor antigens can stimulate innate immunity by sensitizing toll-like receptors and intracellular sensors [25]. DNA vaccines are encumbered by having to cross both cell and nuclear membranes with theoretical concerns for genomic integration and oncologic transformation which have paved the way for RNA vaccines [61]. RNA vaccines can activate the innate immune system by acting as TLR agonists for TLR7 and TLR8 [62-64]. As TLRs are activated, innate immunity is initiated through downstream signaling of $\mathrm{NF}-\mathrm{Kb}$ and production of type I interferons (IFNs) [65]. DAMPs such as heat shock proteins may be incorporated into vaccines to further enhance recruitment of innate immunity while shuttling antigens through the MHC class I pathway to heighten adaptive responses [66, 67]. Our group has shown that preconditioning with tetanus toxoid prior to immunotherapy enhances DC migration to local draining lymph nodes via a CD4+ T cell memory recall response [16, 68-70]. Tetanus boosters mediate recruitment of DCs to draining lymph nodes through a coordinated axis of chemokines including CCL3 and CCL21 mediated in part by these memory $\mathrm{T}$ cells $[16,68-70]$. These data substantiate the interplay between innate immune activation (via tetanus), migration of DCs, and memory $\mathrm{T}$ cells which culminates in enhanced antitumor immunity [16, 68-70]. Recently, systemic RNA delivery encoding for cancer antigens was shown to harness the antiviral defense mechanism [71, 72]. This was shown through systemic IFN alpha dependent activation of APCs and effector cells [71, 72]. The authors propose a mechanism whereby early IFN alpha release from plasmacytoid DCs contributes to migration and maturation of immature DCs while delayed release of IFN alpha from macrophages assists in licensing activated $\mathrm{T}$ cells into fully primed effector cells $[71,72]$. This cross talk between innate and adaptive immunity is requisite for synergy and was corroborated in a phase I dose escalation trial [71, 72]. In this phase I trial, RNA vaccines encoding for four tumor antigens (NY-ESO-1, MAGE-A3, tyrosinase, and TPTE) were well tolerated and elicited dose-dependent release of early IFN $\alpha$ and CXCL10 and de novo T cell immunity against vaccine antigens $[71,72]$. In summary, while peptide vaccines have shown tepid responses in a therapeutic setting with poor correlates for immune activity, RNA vaccines activate innate immune responses and have shown promising effects in preclinical and clinical studies based on enhanced DC migration and maturation.

\section{Immune Checkpoints and Cancer Vaccines}

Checkpoint inhibitors are some of the most promising agents that can be exploited to harness the adaptive immune response against cancer antigens [11, 73, 74]. Immune checkpoints can be targeted with monoclonal antibodies (mAbs) targeting cytotoxic $\mathrm{T}$ lymphocyte associated antigen-4 (CTLA-4) or programmed death-1 (PD-1) which elicit activation of endogenous $\mathrm{T}$ cells against immunogenic epitopes [75]. Ipilimumab, an antagonist of CTLA4 , elicited antitumor $\mathrm{T}$ cell responses in a phase III study for patients with metastatic melanoma improving median overall survival by 3.6 months compared with controls [76]. Ipilimumab enhanced immunity against NY-ESO-1, a cancer/testis antigen expressed in some melanoma patients; NY-ESO-1-seropositive patients with CD8+ T cell responses, as compared to patients with undetectable CD8+ $\mathrm{T}$ cell responses, experienced greater clinical benefit [3]. Subsequent studies have shown that responsiveness to CTLA-4 is increased in patients with higher burdens of nonsynonymous changes, which may result from endogenous $\mathrm{T}$ cell activation against neoantigen epitopes that arise in malignancies with high mutational burdens [77]. Similarly, checkpoint inhibitors antagonizing the PD-1 receptor (nivolumab or pembrolizumab) on $\mathrm{T}$ cells have shown remarkable clinical responses in patients with melanoma and lung cancer [7881]. In patients with advanced nonsquamous non-small cell lung cancer (despite platinum based chemotherapy), the median overall survival was 12.2 months with nivolumab compared to 9.4 months with docetaxel treatment [79]. Like CTLA-4, PD-1 mAbs appear to activate the endogenous T cell response against neoantigens, which may be prospectively identified in the peripheral blood by expression of CD8+PD$1+$ lymphocytes [12, 77, 82, 83]. Since anti-PD-1 and CTLA4 therapies are most effective in patients with evidence for preexisting antitumor immunity, it is likely that these drugs promote established immunity as opposed to inducing de novo responses [84-86]. In a recent study of 46 patients with metastatic melanoma, tumor regression after PD-1 blockade required preexisting $\mathrm{CD} 8+\mathrm{T}$ cells located at the invasive tumor margin that were negatively regulated by the $\mathrm{PD}$ 1 immune inhibitory axis [84]. Since preexisting immunity appears vital for the utility of immune checkpoints, they may have decreased efficacy in cancers with low mutational burdens unless combined with cancer vaccines [87]. Cancer 
vaccines can be used to generate endogenous immunity against tumor antigens, which can be synergistic with checkpoint inhibitors [88]. Immune checkpoint inhibitors can act upon a nascent immunotherapeutic response initiated by cancer vaccines to sustain their proliferation and viability. In preclinical studies, maximal antitumor efficacy hinged on passive and active vaccination in conjunction with anti-PD1 blockade [88]. This combination immunotherapy required a $\mathrm{T}$ cell vaccine, tumor-targeting-antibody, recombinant IL2, and anti-PD-1 blockade, which induced recruitment of tumor infiltrating immune cells and production of intratumoral proinflammatory cytokines in a genetically engineered murine melanoma [88].

Interestingly, the antitumor effects of checkpoint blockade seem to be dependent on distinct species of Bacteroides [89-93]. In murine models and in patients, $T$ cell responses that were specific against $B$. thetaiotaomicron or $B$. fragilis correlated with the effectiveness from CTLA-4 blockade; however, tumors in antibiotic treated mice did not respond to checkpoint inhibition [92]. In preclinical studies, oral Bifidobacterium administration controlled melanoma growth in mice comparably to checkpoint blockade with antiprogrammed death ligand-1 (PD-L1) mAbs [94]. Moreover, the combination of checkpoint blockade and Bifidobacterium nearly eliminated outgrowth of tumor [94]. While these effects are peculiar, the augmented effect of PD-L1 mAb is attributed to enhanced dendritic cell (DC) function by intestinal microbes, enabling heightened CD8+ T cell priming and responsiveness to checkpoint blocking strategies $[91,94]$. Host-innate immunity in the gut is influenced by paneth cells and intestinal DCs which may be responsible for enhancing $\mathrm{T}$ cell immunity induced by checkpoint blockade $[95,96]$. Paneth cells are essential for maintaining host microbial homeostasis and directly recognize enteric bacteria through TLR activation by MyD88 [95]. Intestinal DCs induce selective amounts of IgA protecting against mucosal perturbation and can retain limited amounts of live commensals for days [96, 97]. Given this unique interaction between innate gut and adaptive immunity, cancer vaccines may be harnessed to directly enhance DC function for synergy with immune checkpoint inhibition [95-97]. Similar to how commensal bacteria enhance DCs for synergy with checkpoint blockade, cancer vaccines can be leveraged to directly sensitize host APCs [95-97]. The synergy between intestinal bacteria and checkpoint blockade sheds new light on gut microorganisms and their ability to shape host-innate and adaptive immunity which teeter between proinflammatory and regulatory immune responses [10]. While the exact mechanism responsible for interplay between gut microbiota and potentiation of checkpoint blockade needs to be further elucidated, these data implicate the delicate balance between innate and adaptive immunity which might be molded by cancer vaccines to potentiate antitumor immunity $[85,93]$. In summary, adaptive immunity is maximized when the innate arm is activated in juxtaposition. Attempts to harness both innate and adaptive immunity have demonstrated promising responses both preclinically and clinically. Identifying methods to unlock effective innate immunity while understating its interplay with adaptive immunity promises to enhance both arms of the immune system.

\section{Conclusion}

Cancer vaccines bypass the complexity of cellular therapeutics and activate the innate immune response against cancer antigens, but many of these platforms suffer from inadequate immunogenicity and lack robust antigen specific $\mathrm{T}$ cell responses. Cancer vaccines must overcome the challenges in both their ability to induce the appropriate inflammatory milieu peripherally and their ability to redirect the regulatory stroma intratumorally. To reprogram the peripheral immune milieu, new strategies have been employed to enhance APC recruitment, activation, and trafficking. RNA tumor antigens have been employed to enhance APC recruitment/activation while memory recall responses (using tetanus toxoid) have been shown to enhance APC trafficking. While these advancements appear to directly translate toward improved immunogenicity, potentiating and sustaining these responses remain a significant challenge. To sustain $\mathrm{T}$ cell immunity generated by cancer vaccines, there appears to be a synergistic role for cancer vaccines with immune checkpoint blockade, which works through a delicate balance between the innate and adaptive arms of the immune system. Understanding and manipulating the cross talk between both innate and adaptive arms are likely requisite to engender indelible self-sustaining antitumor immunity. As more effective immunotherapeutics make their way from the bench to the bedside, bridging the gap between the innate and adaptive immunity via novel cancer vaccination platforms is vital to prevent competition and allow for maintenance of a sustained response against cancer antigens.

\section{Competing Interests}

Duane A. Mitchell has patented immunotherapy related technology that have been licensed by Annias Immunotherapeutics, Inc., and Celldex Therapeutics, Inc.

\section{References}

[1] E. F. McCarthy, "The toxins of William B. Coley and the treatment of bone and soft-tissue sarcomas," The Iowa Orthopaedic Journal, vol. 26, pp. 154-158, 2006.

[2] L. Galluzzi, E. Vacchelli, A. Eggermont et al., "Trial watch: experimental Toll-like receptor agonists for cancer therapy," OncoImmunology, vol. 1, no. 5, pp. 699-716, 2012.

[3] J. Yuan, M. Adamow, B. A. Ginsberg et al., "Integrated NYESO-1 antibody and $\mathrm{CD}^{+}{ }^{+} \mathrm{T}$-cell responses correlate with clinical benefit in advanced melanoma patients treated with ipilimumab," Proceedings of the National Academy of Sciences of the United States of America, vol. 108, no. 40, pp. 16723-16728, 2011.

[4] V. Brower, "Approval of provenge seen as first step for cancer treatment vaccines," Journal of the National Cancer Institute, vol. 102, no. 15, pp. 1108-1110, 2010.

[5] P. W. Kantoff, C. S. Higano, N. D. Shore et al., "Sipuleucel-T immunotherapy for castration-resistant prostate cancer," New England Journal of Medicine, vol. 363, no. 5, pp. 411-422, 2010. 
[6] A. A. Alizadeh, V. Aranda, A. Bardelli et al., "Toward understanding and exploiting tumor heterogeneity," Nature Medicine, vol. 21, no. 8, pp. 846-853, 2015.

[7] J. H. Sampson, A. B. Heimberger, G. E. Archer et al., "Immunologic escape after prolonged progression-free survival with epidermal growth factor receptor variant III peptide vaccination in patients with newly diagnosed glioblastoma," Journal of Clinical Oncology, vol. 28, no. 31, pp. 4722-4729, 2010.

[8] P. E. Fecci, A. E. Sweeney, P. M. Grossi et al., "Systemic antiCD25 monoclonal antibody administration safely enhances immunity in murine glioma without eliminating regulatory $\mathrm{T}$ cells," Clinical Cancer Research, vol. 12, no. 14, part 1, pp. 42944305, 2006.

[9] C. A. Learn, P. E. Fecci, R. J. Schmittling et al., "Profiling of $\mathrm{CD}^{+}, \mathrm{CD}^{+}$, and $\mathrm{CD} 4^{+} \mathrm{CD} 25^{+} \mathrm{CD} 45 \mathrm{RO}^{+} \mathrm{FoxP}^{+} \mathrm{T}$ cells in patients with malignant glioma reveals differential expression of the immunologic transcriptome compared with t cells from healthy volunteers," Clinical Cancer Research, vol. 12, no. 24, pp. 7306-7315, 2006

[10] A. Shanker and F. M. Marincola, "Cooperativity of adaptive and innate immunity: implications for cancer therapy," Cancer Immunology, Immunotherapy, vol. 60, no. 8, pp. 1061-1074, 2011.

[11] S. A. Rosenberg, "Raising the bar: the curative potential of human cancer immunotherapy," Science Translational Medicine, vol. 4, no. 127, 2012.

[12] N. A. Rizvi, M. D. Hellmann, A. Snyder et al., "Mutational landscape determines sensitivity to PD-1 blockade in non-small cell lung cancer," Science, vol. 348, no. 6230, pp. 124-128, 2015.

[13] C. D. Pham, C. Flores, C. Yang et al., "Differential immune microenvironments and response toimmunecheckpoint blockadeamong molecular subtypes of murine medulloblastoma," Clinical Cancer Research, vol. 22, no. 3, pp. 582-595, 2016.

[14] A. P. Rapoport, E. A. Stadtmauer, G. K. Binder-Scholl et al., "NY-ESO-1-specific TCR-engineered T cells mediate sustained antigen-specific antitumor effects in myeloma," Nature Medicine, vol. 21, no. 8, pp. 914-921, 2015.

[15] C. S. Cobbs, "Cytomegalovirus and brain tumor: epidemiology, biology and therapeutic aspects," Current Opinion in Oncology, vol. 25, no. 6, pp. 682-688, 2013.

[16] D. A. Mitchell, K. A. Batich, M. D. Gunn et al., "Tetanus toxoid and CCL3 improve dendritic cell vaccines in mice and glioblastoma patients," Nature, vol. 519, no. 7543, pp. 366-369, 2015.

[17] H. Jähnisch, S. Füssel, A. Kiessling et al., "Dendritic cell-based immunotherapy for prostate cancer," Clinical and Developmental Immunology, vol. 2010, Article ID 517493, 8 pages, 2010.

[18] C. J. Desmet and K. J. Ishii, "Nucleic acid sensing at the interface between innate and adaptive immunity in vaccination," Nature Reviews Immunology, vol. 12, no. 7, pp. 479-491, 2012.

[19] A. M. Hicks, G. Riedlinger, M. C. Willingham et al., "Transferable anticancer innate immunity in spontaneous regression/complete resistance mice," Proceedings of the National Academy of Sciences of the United States of America, vol. 103, no. 20, pp. 7753-7758, 2006.

[20] Y. A. Yu, C. Galanis, Y. Woo et al., "Regression of human pancreatic tumor xenografts in mice after a single systemic injection of recombinant vaccinia virus GLV-1h68," Molecular Cancer Therapeutics, vol. 8, no. 1, pp. 141-151, 2009.

[21] A. M. Hicks, M. C. Willingham, W. Du, C. S. Pang, L. J. Old, and Z. Cui, "Effector mechanisms of the anti-cancer immune responses of macrophages in SR/CR mice," Cancer immunity: a journal of the Academy of Cancer Immunology, vol. 6, 2006.
[22] P. Matzinger, "The danger model: a renewed sense of self," Science, vol. 296, no. 5566, pp. 301-305, 2002.

[23] P. Matzinger, "The evolution of the danger theory," Expert Review of Clinical Immunology, vol. 8, no. 4, pp. 311-317, 2012.

[24] P. M. Barral, D. Sarkar, Z.-Z. Su et al., "Functions of the cytoplasmic RNA sensors RIG-I and MDA-5: key regulators of innate immunity," Pharmacology and Therapeutics, vol. 124, no. 2, pp. 219-234, 2009.

[25] R. Barbalat, S. E. Ewald, M. L. Mouchess, and G. M. Barton, "Nucleic acid recognition by the innate immune system," Annual Review of Immunology, vol. 29, pp. 185-214, 2011.

[26] T. Aoshi, S. Koyama, K. Kobiyama, S. Akira, and K. J. Ishii, "Innate and adaptive immune responses to viral infection and vaccination," Current Opinion in Virology, vol. 1, no. 4, pp. 226232, 2011.

[27] P. Ponsaerts, V. F. I. Van Tendeloo, and Z. N. Berneman, "Cancer immunotherapy using RNA-loaded dendritic cells," Clinical and Experimental Immunology, vol. 134, no. 3, pp. 378-384, 2003.

[28] S. K. Nair, A. Heiser, D. Boczkowski et al., "Induction of cytotoxic $\mathrm{T}$ cell responses and tumor immunity against unrelated tumors using telomerase reverse transcriptase RNA transfected dendritic cells," Nature Medicine, vol. 6, no. 9, pp. 1011-1017, 2000.

[29] D. Boczkowski, S. K. Nair, D. Snyder, and E. Gilboa, "Dendritic cells pulsed with RNA are potent antigen-presenting cells in vitro and in vivo," Journal of Experimental Medicine, vol. 184, no. 2, pp. 465-472, 1996.

[30] S. A. Rosenberg and M. E. Dudley, "Cancer regression in patients with metastatic melanoma after the transfer of autologous antitumor lymphocytes," Proceedings of the National Academy of Sciences of the United States of America, vol. 101, supplement 2, pp. 14639-14645, 2004.

[31] L. Gattinoni, S. E. Finkelstein, C. A. Klebanoff et al., "Removal of homeostatic cytokine sinks by lymphodepletion enhances the efficacy of adoptively transferred tumor-specific CD8+ T cells," Journal of Experimental Medicine, vol. 202, no. 7, pp. 907-912, 2005.

[32] A. S. Margol, N. J. Robison, J. Gnanachandran et al., "Tumorassociated macrophages in $\mathrm{SHH}$ subgroup of medulloblastomas," Clinical Cancer Research, vol. 21, no. 6, pp. 1457-1465, 2015.

[33] X. Feng, B. Li, H. Ye, and D. Long, "Increased frequency of $\mathrm{CD} 4{ }^{+} \mathrm{CD} 25^{\text {high }} \mathrm{FoxP}^{+}$regulatory $\mathrm{T}$ cells in patients with hepatocellular carcinoma," Archivum Immunologiae et Therapiae Experimentalis, vol. 59, no. 4, pp. 309-314, 2011.

[34] K. N. Kodumudi, A. Weber, A. A. Sarnaik, and S. Pilon-Thomas, "Blockade of myeloid-derived suppressor cells after induction of lymphopenia improves adoptive $\mathrm{T}$ cell therapy in a murine model of melanoma," The Journal of Immunology, vol. 189, no. 11, pp. 5147-5154, 2012.

[35] M. J. Welters, T. C. van der Sluis, H. van Meir et al., "Vaccination during myeloid cell depletion by cancer chemotherapy fosters robust T cell responses," Science Translational Medicine, vol. 8, no. 334, Article ID 334ra52, 2016.

[36] A. Iwasaki and R. Medzhitov, "Toll-like receptor control of the adaptive immune responses," Nature Immunology, vol. 5, no. 10, pp. 987-995, 2004.

[37] S. Ito, C. M. Bollard, M. Carlsten et al., "Ultra-low dose interleukin-2 promotes immune-modulating function of regulatory $\mathrm{t}$ cells and natural killer cells in healthy volunteers," Molecular Therapy, vol. 22, no. 7, pp. 1388-1395, 2014. 
[38] C. J. M. Bell, Y. Sun, U. M. Nowak et al., "Sustained in vivo signaling by long-lived IL-2 induces prolonged increases of regulatory T cells," Journal of Autoimmunity, vol. 56, pp. 66-80, 2015.

[39] J. Koreth, H. T. Kim, K. T. Jones et al., "Efficacy, durability, and response predictors of low-dose interleukin-2 therapy for chronic graft-versus-host disease," Blood, vol. 128, no. 1, pp. 130137, 2016.

[40] C. Onyilagha, I. Okwor, S. Kuriakose, R. Singh, and J. Uzonna, "Low-dose intradermal infection with Trypanosoma congolense leads to expansion of regulatory $\mathrm{T}$ cells and enhanced susceptibility to reinfection," Infection and Immunity, vol. 82, no. 3, pp. 1074-1083, 2014.

[41] O. M. Grauer, S. Nierkens, E. Bennink et al., "CD4+FoxP3+ regulatory $\mathrm{T}$ cells gradually accumulate in gliomas during tumor growth and efficiently suppress antiglionia immune responses in vivo," International Journal of Cancer, vol. 121, no. 1, pp. 95-105, 2007.

[42] S. L. Highfill, Y. Cui, A. J. Giles et al., "Disruption of CXCR2mediated MDSC tumor trafficking enhances anti-PD1 efficacy," Science Translational Medicine, vol. 6, no. 237, Article ID 237ra67, 2014.

[43] F. J. Kohlhapp and H. L. Kaufman, "Molecular pathways: mechanism of action for talimogene laherparepvec, a new oncolytic virus immunotherapy," Clinical Cancer Research, vol. 22, no. 5, pp. 1048-1054, 2016.

[44] H. L. Kaufman, F. J. Kohlhapp, and A. Zloza, "Oncolytic viruses: a new class of immunotherapy drugs," Nature Reviews Drug Discovery, vol. 14, no. 9, pp. 642-662, 2015.

[45] B. R. G. Williams, "PKR; a sentinel kinase for cellular stress," Oncogene, vol. 18, no. 45, pp. 6112-6120, 1999.

[46] T. Kawai and S. Akira, "Innate immune recognition of viral infection," Nature Immunology, vol. 7, no. 2, pp. 131-137, 2006.

[47] M. J. Scanlan, A. O. Gure, A. A. Jungbluth, L. J. Old, and Y.-T. Chen, "Cancer/testis antigens: an expanding family of targets for cancer immunotherapy," Immunological Reviews, vol. 188, pp. 22-32, 2002.

[48] M. F. Gjerstorff, M. H. Andersen, and H. J. Ditzel, "Oncogenic cancer/testis antigens: prime candidates for immunotherapy," Oncotarget, vol. 6, no. 18, pp. 15772-15787, 2015.

[49] O. L. Caballero and Y.-T. Chen, "Cancer/testis (CT) antigens: potential targets for immunotherapy," Cancer Science, vol. 100, no. 11, pp. 2014-2021, 2009.

[50] J. H. Sampson, K. D. Aldape, G. E. Archer et al., "Greater chemotherapy-induced lymphopenia enhances tumor-specific immune responses that eliminate EGFRvIII-expressing tumor cells in patients with glioblastoma," Neuro-Oncology, vol. 13, no. 3, pp. 324-333, 2011.

[51] R. Babu and D. C. Adamson, "Rindopepimut: an evidencebased review of its therapeutic potential in the treatment of EGFRvIII-positive glioblastoma," Core Evidence, vol. 7, pp. 93103, 2012.

[52] C. M. Solbrig, J. K. Saucier-Sawyer, V. Cody, W. M. Saltzman, and D. J. Hanlon, "Polymer nanoparticles for immunotherapy from encapsulated tumor-associated antigens and whole tumor cells," Molecular Pharmaceutics, vol. 4, no. 1, pp. 47-57, 2007.

[53] G. Shi, C. Zhang, R. Xu et al., "Enhanced antitumor immunity by targeting dendritic cells with tumor cell lysate-loaded chitosan nanoparticles vaccine," Biomaterials, vol. 113, pp. 191-202, 2017.
[54] J. Schuster, R. K. Lai, L. D. Recht et al., "A phase II, multicenter trial of rindopepimut (CDX-110) in newly diagnosed glioblastoma: the ACT III study," Neuro-Oncology, vol.17, no. 6, pp. 854861,2015

[55] N. A. Sheikh, D. Petrylak, P. W. Kantoff et al., "Sipuleucel$\mathrm{T}$ immune parameters correlate with survival: an analysis of the randomized phase 3 clinical trials in men with castrationresistant prostate cancer," Cancer Immunology, Immunotherapy, vol. 62, no. 1, pp. 137-147, 2013.

[56] A. Yang, E. Farmer, T. C. Wu, and C. Hung, "Perspectives for therapeutic HPV vaccine development," Journal of Biomedical Science, vol. 23, no. 1, p. 75, 2016.

[57] L. A. Johnson and J. H. Sampson, "Immunotherapy approaches for malignant glioma from 2007 to 2009," Current Neurology and Neuroscience Reports, vol. 10, no. 4, pp. 259-266, 2010.

[58] I. F. Pollack, R. I. Jakacki, L. H. Butterfield et al., "Antigenspecific immune responses and clinical outcome after vaccination with glioma-associated antigen peptides and polyinosinicpolycytidylic acid stabilized by lysine and carboxymethylcellulose in children with newly diagnosed malignant brainstem and nonbrainstem gliomas," Journal of Clinical Oncology, vol. 32, no. 19, pp. 2050-2058, 2014.

[59] D. A. Mitchell and S. K. Nair, "RNA-transfected dendritic cells in cancer immunotherapy," The Journal of Clinical Investigation, vol. 106, no. 9, pp. 1065-1069, 2000.

[60] D. A. Mitchell and S. K. Nair, "RNA transfected dendritic cells as cancer vaccines," Current Opinion in Molecular Therapeutics, vol. 2, no. 2, pp. 176-181, 2000.

[61] J. B. Ulmer, P. W. Mason, A. Geall, and C. W. Mandl, "RNAbased vaccines," Vaccine, vol. 30, no. 30, pp. 4414-4418, 2012.

[62] M. Fotin-Mleczek, K. M. Duchardt, C. Lorenz et al., "Messenger RNA-based vaccines with dual activity induce balanced TLR-7 dependent adaptive immune responses and provide antitumor activity," Journal of Immunotherapy, vol. 34, no. 1, pp. 1-15, 2011.

[63] A. L. Blasius and B. Beutler, "Intracellular toll-like receptors," Immunity, vol. 32, no. 3, pp. 305-315, 2010.

[64] S. S. Diebold, T. Kaisho, H. Hemmi, S. Akira, and C. Reis E Sousa, "Innate antiviral responses by means of TLR7-mediated recognition of single-stranded RNA," Science, vol. 303, no. 5663, pp. 1529-1531, 2004.

[65] M. H. Nelson, J. S. Bowers, S. R. Bailey et al., "Toll-like receptor agonist therapy can profoundly augment the antitumor activity of adoptively transferred $\mathrm{CD}^{+} \mathrm{T}$ cells without host preconditioning," Journal for Immuno Therapy of Cancer, vol. 4, article 6, 2016.

[66] L. Sanchez-Perez, T. Kottke, G. A. Daniels et al., "Killing of normal melanocytes, combined with heat shock protein 70 and CD40L expression, cures large established melanomas," The Journal of Immunology, vol. 177, no. 6, pp. 4168-4177, 2006.

[67] J. Kunisawa and N. Shastri, "Hsp90 $\alpha$ chaperones large Cterminally extended proteolytic intermediates in the MHC class I antigen processing pathway," Immunity, vol. 24, no. 5, pp. 523534, 2006.

[68] R. L. Sabado and N. Bhardwaj, "Cancer immunotherapy: dendritic-cell vaccines on the move," Nature, vol. 519, no. 7543, pp. 300-301, 2015.

[69] S. Seton-Rogers, "Cancer immunotherapy: primed for a response," Nature Reviews Drug Discovery, vol. 14, no. 5, article no. 312, 2015.

[70] K. A. Batich, A. M. Swartz, and J. H. Sampson, "Preconditioning vaccine sites for mRNA-transfected dendritic cell therapy and 
antitumor efficacy," Methods in Molecular Biology, vol. 1403, pp. 819-838, 2016.

[71] L. M. Kranz, M. Diken, H. Haas et al., "Systemic RNA delivery to dendritic cells exploits antiviral defence for cancer immunotherapy," Nature, vol. 534, no. 7607, pp. 396-401, 2016.

[72] J. De Vries and C. Figdor, "Immunotherapy: cancer vaccine triggers antiviral-Type defences," Nature, vol. 534, no. 7607, pp. 329-331, 2016.

[73] S. L. Topalian, F. S. Hodi, J. R. Brahmer et al., "Safety, activity, and immune correlates of anti-PD-1 antibody in cancer," The New England Journal of Medicine, vol. 366, no. 26, pp. 24432454, 2012.

[74] N. P. Restifo, M. E. Dudley, and S. A. Rosenberg, "Adoptive immunotherapy for cancer: harnessing the T cell response," Nature Reviews Immunology, vol. 12, no. 4, pp. 269-281, 2012.

[75] T. Okazaki, S. Chikuma, Y. Iwai, S. Fagarasan, and T. Honjo, "A rheostat for immune responses: the unique properties of PD-1 and their advantages for clinical application," Nature Immunology, vol. 14, no. 12, pp. 1212-1218, 2013.

[76] F. S. Hodi, S. J. O’Day, D. F. McDermott et al., "Improved survival with ipilimumab in patients with metastatic melanoma," The New England Journal of Medicine, vol. 363, no. 8, pp. 711723, 2010.

[77] A. Snyder, V. Makarov, T. Merghoub et al., "Genetic basis for clinical response to CTLA-4 blockade in melanoma," New England Journal of Medicine, vol. 371, no. 23, pp. 2189-2199, 2014.

[78] S. M. Ansell, A. M. Lesokhin, I. Borrello et al., "PD-1 blockade with nivolumab in relapsed or refractory Hodgkin's lymphoma," The New England Journal of Medicine, vol. 372, no. 4, pp. 311-319, 2015.

[79] H. Borghaei, L. Paz-Ares, L. Horn et al., "Nivolumab versus docetaxel in advanced nonsquamous non-small-cell lung cancer," New England Journal of Medicine, vol. 373, no. 17, pp. 1627-1639, 2015.

[80] J. R. Brahmer, S. S. Tykodi, L. Q. M. Chow et al., "Safety and activity of anti-PD-L1 antibody in patients with advanced cancer," The New England Journal of Medicine, vol. 366, no. 26, pp. 2455-2465, 2012.

[81] J. Duraiswamy, K. M. Kaluza, G. J. Freeman, and G. Coukos, "Dual blockade of PD-1 and CTLA-4 combined with tumor vaccine effectively restores T-cell rejection function in tumors," Cancer Research, vol. 73, no. 12, pp. 3591-3603, 2013.

[82] Y. C. Lu and P. F. Robbins, “Targeting neoantigens for cancer immunotherapy," International Immunology, vol. 28, no. 7, pp. 365-370, 2016.

[83] A. Gros, M. R. Parkhurst, E. Tran et al., "Prospective identification of neoantigen-specific lymphocytes in the peripheral blood of melanoma patients," Nature Medicine, vol. 22, no. 4, pp. 433438, 2016.

[84] P. C. Tumeh, C. L. Harview, J. H. Yearley et al., "PD-1 blockade induces responses by inhibiting adaptive immune resistance," Nature, vol. 515, no. 7528, pp. 568-571, 2014.

[85] N. R. West and F. Powrie, "Immunotherapy not working? Check your microbiota," Cancer Cell, vol. 28, no. 6, pp. 687-689, 2015.

[86] R.-R. Ji, S. D. Chasalow, L. Wang et al., "An immune-active tumor microenvironment favors clinical response to ipilimumab," Cancer Immunology, Immunotherapy, vol. 61, no. 7, pp. 1019-1031, 2012.

[87] C. Kandoth, M. D. McLellan, F. Vandin et al., "Mutational landscape and significance across 12 major cancer types," Nature, vol. 502, no. 7471, pp. 333-339, 2013.
[88] K. D. Moynihan, C. F. Opel, G. L. Szeto et al., "Eradication of large established tumors in mice by combination immunotherapy that engages innate and adaptive immune responses," Nature Medicine, vol. 22, no. 12, pp. 1402-1410, 2016.

[89] C. J. M. Melief, "Cancer: immune pact with the enemy," Nature, vol. 450, no. 7171, pp. 803-804, 2007.

[90] C. M. Koebel, W. Vermi, J. B. Swann et al., "Adaptive immunity maintains occult cancer in an equilibrium state," Nature, vol. 450, no. 7171, pp. 903-907, 2007.

[91] A. Snyder, E. Pamer, and J. Wolchok, "Could microbial therapy boost cancer immunotherapy?” Science, vol. 350, no. 6264, pp. 1031-1032, 2015.

[92] M. Vétizou, J. M. Pitt, R. Daillère et al., "Anticancer immunotherapy by CTLA-4 blockade relies on the gut microbiota," Science, vol. 350, no. 6264, pp. 1079-1084, 2015.

[93] G. K. Alderton, "Tumour immunology: intestinal bacteria are in command," Nature Reviews Immunology, vol. 16, no. 1, 2016.

[94] A. Sivan, L. Corrales, N. Hubert et al., "Commensal Bifidobacterium promotes antitumor immunity and facilitates anti-PDL1 efficacy," Science, vol. 350, no. 6264, pp. 1084-1089, 2015.

[95] S. Vaishnava, C. L. Behrendt, A. S. Ismail, L. Eckmann, and L. V. Hooper, "Paneth cells directly sense gut commensals and maintain homeostasis at the intestinal host-microbial interface," Proceedings of the National Academy of Sciences of the United States of America, vol. 105, no. 52, pp. 20858-20863, 2008.

[96] A. J. Macpherson and T. Uhr, "Induction of protective IgA by intestinal dendritic cells carrying commensal bacteria," Science, vol. 303, no. 5664, pp. 1662-1665, 2004.

[97] J.-P. Kraehenbuhl and M. Corbett, "Keeping the gut microflora at bay," Science, vol. 303, no. 5664, pp. 1624-1625, 2004. 


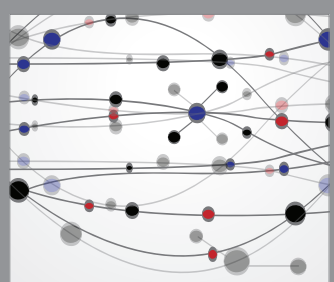

The Scientific World Journal
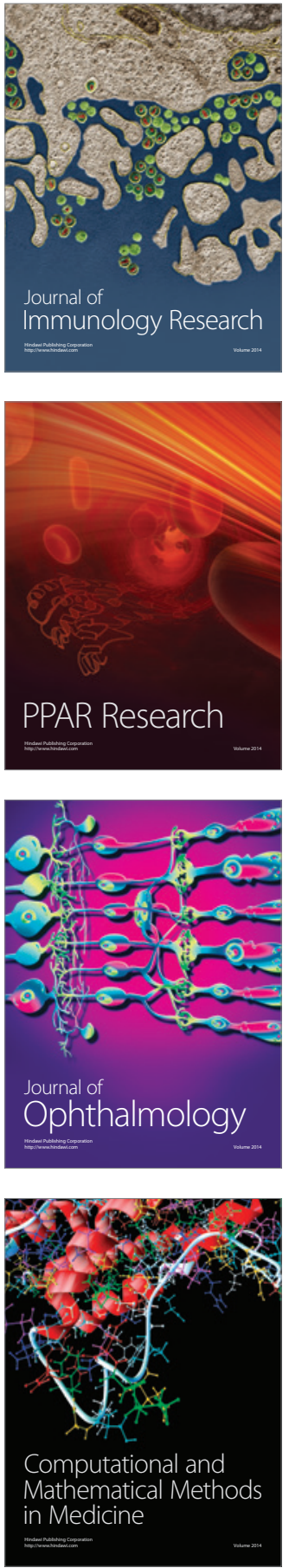

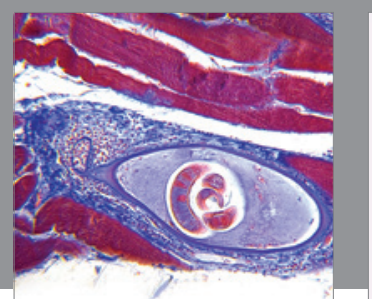

Gastroenterology Research and Practice
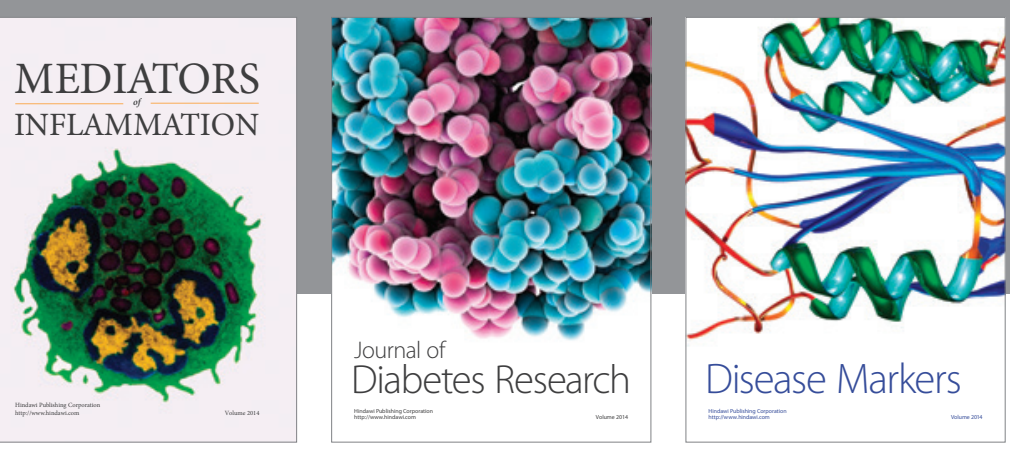

Disease Markers

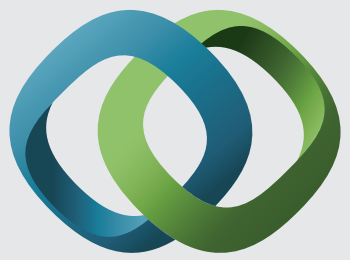

\section{Hindawi}

Submit your manuscripts at

https://www.hindawi.com
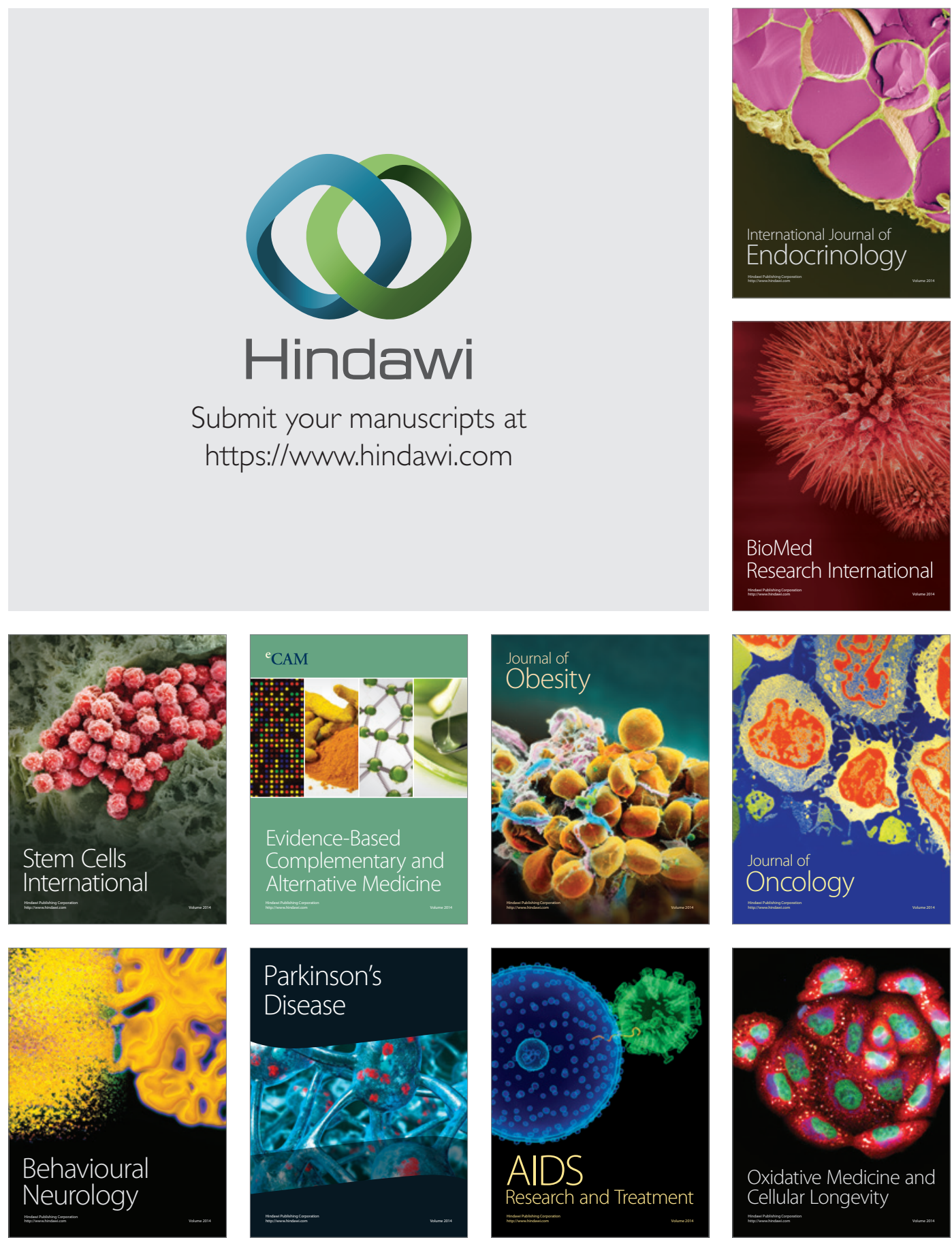